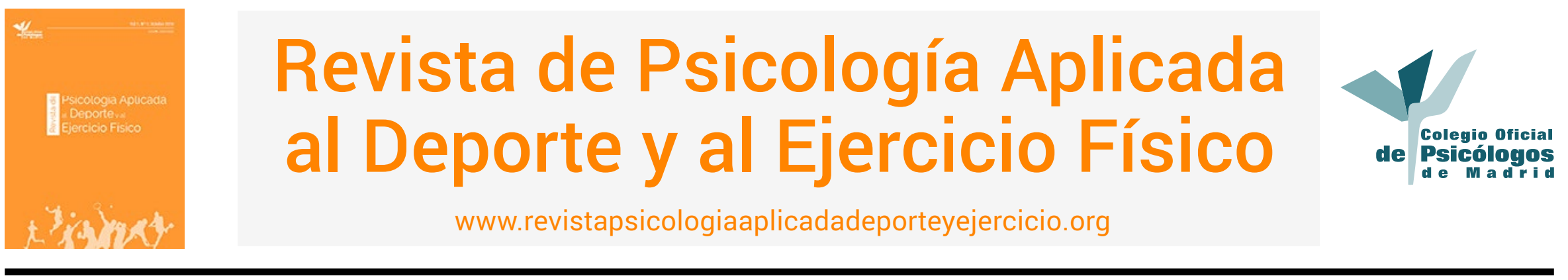

\title{
El perfil profesional del especialista en psicología del deporte en fútbol
}

\author{
David Peris-Delcampo y Enrique Cantón Chirivella \\ Universitat de València, España
}

RESUMEN: En el fútbol actual es cada vez más frecuente la incorporación de distintos profesionales para realizar un trabajo especializado, a través de diferentes metodologías, y donde el psicólogo/a experto en psicología del deporte aplicado al fútbol tiene que ser el verdadero referente del entrenamiento mental en este deporte. Para ello, es necesario que desarrolle una serie de competencias, adaptadas al entorno específico del fútbol y aplicar una serie de principios de actuación que hagan que, con su trabajo, se incorpore esta figura profesional de manera habitual como un agente más en el cada vez más profesionalizado y especializado mundo del fútbol. En este trabajo, se recogen los principales aspectos formativos y de experiencia del psicólogo/a del fútbol, entendiendo que actualmente existe una acreditación profesional del psicólogo del deporte y que sería muy conveniente la sub-especialización como psicólogo/a del fútbol. En base a ello se describen las competencias profesionales del psicólogo/a del fútbol y se proponen una serie de aspectos clave del psicólogo/a para que realice un trabajo eficiente en este deporte tan característico.

PALABRAS CLAVES: Intervención psicológica, fútbol, rol profesional, psicólogo.

\section{The professional profile of the specialist in sports psychology in football (soccer)}

ABSTRACT: In today's football/soccer, incorporation of different professionals to perform specialized work through different methodologies is increasingly common, and psychologists/experts in sports psychology applied to football have to be the true reference for mental training in this sport. To do this, they need to acuire a number of skills adapted to the specific environment of football and apply a series of principles of action that, with their work, allow for this professional figure to regularly become one more actor in the increasingly professionalized and specialized world of soccer/football. This article discusses the main training and experience requirements of football psychologists, understanding that there is currently a professional accreditation as sports psychologist and that it would be convenient to sub-specialize as a football psychologist. Based on this, the professional competences of soccer psychologists are described and a number of aspects essential for the psychologist to perform an efficient job in this characteristic sport are proposed.

KEYWORDS: Psychological intervention, football/soccer, professional role, psychologist.

\section{O perfil profissional do especialista em psicologia desportiva no futebol}

RESUMO: No futebol de hoje, é cada vez mais comum a incorporação de diferentes profissionais para realizar trabalhos especializados, através de diferentes metodologias, e onde o psicólogo/a especialista em psicologia desportiva aplicada ao futebol tem de ser a verdadeira referência do treino mental neste desporto. Para tal, é necessário desenvolver várias competências, adaptadas ao ambiente específico do futebol e aplicar uma série de princípios de ação que levem a que, com o seu trabalho, se incorpore esta figura profissional numa base regular como mais um agente no mundo do futebol que é cada vez mais profissionalizado e especializado. Neste trabalho, reúnem-se os principais aspetos formativos e de experiência do/a psicólogo/a de futebol, entendendo-se que atualmente existe uma certificação profissional do/a psicólogo/a desportivo/a e que seria muito relevante a subespecialização como psicólogo de futebol.

\footnotetext{
David Peris-Delcampo es Vicepresidente de la Federación Española de Psicología del Deporte; Enrique Cantón Chirivella es Coordinador General de la División de Psicología del Deporte del Consejo General de Psicología de España.

Para la correspondencia de este artículo enviar a David Peris Delcampo. Avda. Jaume I, nº 26, $7^{\circ} \mathrm{H}, 12530$ Burriana (Castellón), España.

E-mail: davidperisdelcampo@hotmail.com
} 
Com base nisso, descrevem-se as competências profissionais do/a psicólogo/a de futebol e propõe-se uma série de aspetos chave do/a psicólogo/a para que possa realizar um trabalho eficiente neste desporto tão característico.

PALAVRAS-CHAVE: Intervenção psicológica, futebol, papel profissional, psicólogo.

Artículo recibido: 04/03/2018 | Artículo aceptado: 20/05/2018

La intervención psicológica en el deporte y en especial en el fútbol es, cada vez más, una demanda normalizada. Ha llegado con posterioridad a la aplicación de otros conocimientos científicos desde otras áreas, como la medicina, la preparación física u otros aspectos técnico-tácticos. La parte psicológica del entrenamiento deportivo es quizás la que, siendo más reciente, más rápidamente está siendo incorporada para ayudar a controlar de manera eficiente todos los aspectos del entrenamiento en el fútbol.

La Psicología del Deporte en el fútbol es relativamente reciente en España a pesar de que hubo experiencias muy exitosas de intervención psicológica en este deporte, como la realizada por Jesús García-Barrero y Rosana Llames (1992) con la Selección Olímpica de Fútbol en Barcelona'92, si bien quizás otras no fueron demasiado afortunadas, a lo que cabe añadir la escasez inicial de psicólogos/as formados adecuadamente en la especialidad, o la falta de capacitación profesional de muchos técnicos (véase Olmedilla, García y Garcés de Los Fayos, 1998) y directivos del deporte, que no entendían esta labor o la consideraban suplible por las tareas de otros con alguna escueta noción sobre ello, como ya ocurriera con la preparación física. Estas y seguramente alguna razón más de índole económica y social, pueden explicar porque el trabajo del psicólogo/a en el fútbol tuvo una cierta "demora" en incorporarse de manera normalizada y en ocasiones, también el porqué de cierta reticencia a contar con éstos profesionales (Cantón, 2010b).

La evolución de la figura profesional del psicólogo/a del deporte necesita para su comprensión, el hacer referencia a la eficacia progresivamente demostrada en las diferentes intervenciones psicológicas en fútbol y, sobre todo, en la labor desempeñada en la base, con la iniciación deportiva y tecnificación de las escuelas de este deporte (García-Naveira y Jerez, 2012; Morilla et al., 2003; Navarrón, Godoy-Izquierdo, Vélez, Ramírez-Molina y Jiménez-Torres, 2017). Además, en el fútbol ha habido una mejora de la cualificación y formación de sus técnicos en otros muchos aspectos, lo que indirectamente ha favorecido la progresiva incorporación de la Psicología del Deporte, desarrollándose una gran evolución de la propia metodología de trabajo en éste. A groso modo, podemos entender que en un primer momento prevalecía la metodología analítica, en la que se diferenciaban las diferentes partes del entrenamiento "por separado", distinguiendo claramente cada una de ellas en fragmentos de las sesiones de trabajo y donde cada una tiene su parte del tiempo en el entrenamiento. Un libro clásico en este sentido y que marcó una época en el campo de la preparación física aplicada a este deporte es el de Álvarez del Villar (1985), donde se plantea el trabajo de aspectos condicionales ("físicos") de manera diferenciada y "a parte" de los otros apartados del fútbol (como por ejemplo el técnico, el táctico o el estratégico). Desde este planteamiento, el trabajo psicológico también lo realizaría una persona especializada independientemente, aunque vinculada, con los otros aspectos del entrenamiento.

Posteriormente y gracias a la importante aportación de autores como Seirullo (1999), se introdujo la metodología integrada o globalizada, donde las diferentes partes de entrenamiento en el fútbol (fundamentalmente el técnico, táctico, físico y, en alguna ocasión el psicológico) se "juntan" en un mismo ejercicio de entrenamiento para realizar un trabajo mucho más cercano a lo que sería una situación real de juego. Incluso en muchos casos, teniendo en cuenta otros aspectos del jugador de forma global, como el socioafectivo, creativo, cognitivo o emocional, aspectos todos de orden psicológico.

Un elemento central de la evolución del entrenamiento globalizado es, precisamente, que se basa en la especificidad, enfoque en el que quizás el modelo más conocido sea la periodización táctica (Tamarit, 2013). En ésta, se entiende al fútbol como un juego, como un todo, donde no existen partes diferenciadas y sí elementos interconectados entre sí, entendiendo que manejar un aspecto determinado influye en los demás. Desde esta perspectiva no hay partes, hay un todo donde cada aspecto trabajado se retroalimenta con los demás. En este sentido, ya es fácil contemplar cómo sobre todo en el fútbol base, se busca que sea el propio jugador el que entienda el juego y la metodología va encaminada a que descubra y soluciones situaciones, a través de un entorno de descubrimiento diseñado por los entrenadores y demás técnicos, entendido como descubrimiento guiado o coaching (Peris-Delcampo, 2016).

Con el progresivo aumento de la tecnificación y la mejora de la cualificación científıca y aplicada desde las diferentes disciplinas científicas, así como una mayor comprensión de la relevancia de los factores psicológicos y, sin duda, debido 
a los resultados prácticos que se han venido produciendo, la incorporación de esta figura profesional ya es un hecho, al margen de que queda un camino por recorrer en cuanto a las condiciones formales, económicas y sociales de su incorporación. También está contribuyendo a su establecimiento dentro del fútbol el que, a nivel general, en el deporte y el ejercicio, se van estableciendo con claridad las competencias profesionales que la definen, con su correlato formativo especializado y su reconcomiendo por parte de organizaciones profesionales, científicas y políticas (Cantón, 2016).

El presente trabajo tiene como objetivo principal determinar los aspectos fundamentales a tener en cuenta por el Psicólogo Experto en Psicología del Deporte (en adelante PEPD) para que realice su trabajo como uno de los profesionales habituales dentro de los equipos técnicos u organigramas en el fútbol en cualquiera de sus posibilidades; y sea reconocido por los demás agentes de este deporte como realmente el único profesional cualificado de la preparación "mental" en el fútbol (Cantón, 2010a; Peris-Delcampo, 2017a, 2017b).

\section{Proceso formativo del psicólogo/a del deporte aplicado al futbol}

El fútbol, como sabemos, es un deporte con una serie de demandas características a las que la Psicología del Deporte debe dar respuesta de manera óptima. Ahora bien, ¿qué perfil debería tener un psicólogo/a dedicado al fútbol? Des- de nuestro punto de vista, al menos tendría que cumplir los requisitos señalados desde el Consejo General de la Psicología de España (CGPE), en cuanto a formación y experiencia práctica, además de mostrar las competencias propias del psicólogo del deporte (relacionados con el saber hacer). También, para según qué puesto de responsabilidad y desde luego para ser reconocido formalmente como Experto en Psicología del Deporte, debe tener una cierta experiencia en el trabajo previo en diferentes equipos o clubes. Es decir, además de contar con el Grado/Licenciatura en Psicología, debe saber de psicología aplicada al deporte y después entender las necesidades específicas del fútbol para saber adaptarse a ellas optimizando su trabajo.

La División de Psicología del Deporte del CGPE (PACFD) ha desarrollado un procedimiento de acreditación profesional del psicólogo/a del deporte, con unos criterios claramente establecidos basados en una formación en Psicología, después en Psicología del Deporte y con una experiencia reconocida en esta especialidad (CGPE, 2018). Recogiendo estos criterios y entendiendo además que cualquier profesional de la psicología que trabaje en deporte debería cumplirlos, entendemos además que PEPD que trabaje en fútbol debería de cumplir unos requisitos específicos para cubrir las necesidades de este deporte. En la tabla 1 resumimos una propuesta de requerimientos que debería cumplir un profesional de la psicología en el fútbol, teniendo en cuenta los planteados por PACFD para la acreditación en PEPD y los "extra" que debería cumplir un/a psicólogo/a del fútbol.

\section{Tabla 1. Propuesta de requisitos del Profesional de la Psicología del Deporte Aplicado al Fútbol}

\begin{tabular}{ll} 
Requisitos previos & $\begin{array}{l}\text { Estar habilitado profesionalmente para el ejercicio profesional de la psicología, a través de una formación } \\
\text { académica reglada (tener Licenciatura o Grado en psicología). }\end{array}$ \\
\hline Requisitos colegiales & $\begin{array}{l}\text { Estar colegiado en algunos de los Colegios Oficiales de Psicología de España. Estar al corriente de pago de } \\
\text { cuota colegial y no tener sanción deontológica. Estar inscrito en la División de Psicología del Deporte del Con- } \\
\text { sejo General de la Psicología de España. }\end{array}$ \\
\hline $\begin{array}{l}\text { Formación específica en Psico- } \\
\text { logía del Deporte }\end{array}$ & $\begin{array}{l}\text { Tener una formación específica en Psicología del Deporte, que actualmente se oferta en diversos cursos de } \\
\text { postgrado de diferentes universidades o colegios oficiales reconocidos, en España. Al menos, según los crite- } \\
\text { rios de PACFD, un mínimo de } 400 \text { horas acreditadas de formación reconocida especializada. }\end{array}$ \\
\hline $\begin{array}{l}\text { Experiencia aplicada } \\
\text { Conocimientos mínimos del } \\
\text { deporte del fútbol }\end{array}$ & $\begin{array}{l}\text { Tener una experiencia demostrada dentro del deporte aplicando la psicología, en cualquiera de sus posibilida- } \\
\text { des (al menos, según los criterios de PACFD), un mínimo de } 4 \text { años de experiencia profesional. }\end{array}$ \\
\hline $\begin{array}{l}\text { Experiencia profesional } \\
\text { cular de este deporte. }\end{array}$ & $\begin{array}{l}\text { Tener una experiencia necesaria para actuar en diferentes contextos relacionados con el fútbol, que será más } \\
\text { necesaria cuando la complejidad del entorno exija una mayor calidad del trabajo de intervención psicológica. }\end{array}$ \\
\hline $\begin{array}{l}\text { Competencias específicas del } \\
\text { psicólogo aplicado al fútbol }\end{array}$ & $\begin{array}{l}\text { Desarrollar las competencias profesionales generales de Psicología de los deportes adaptados a los diferentes } \\
\text { entornos de aplicación en el fútbol. }\end{array}$
\end{tabular}


Actualmente, ya es posible acreditarse como Psicólogo/a Experto en Psicología del Deporte (PEPD), de manera general. No obstante, pensando en la necesaria adaptabilidad del trabajo psicológico a un deporte como el fútbol, consideramos necesario que PEPD que trabaje en este campo sepa además cómo desenvolverse en este medio, por lo que es necesario que adquiera una serie de competencias específicas para poder realizar eficientemente su trabajo.

Es necesario, también, tener en cuenta que el proceso de acreditación lleva un tiempo, por lo que habrá psicólogos/as que estén en periodo de obtención de ese proceso que, aunque no cumplan la totalidad de los requisitos para obtener la acreditación PEPD (por ejemplo la experiencia) sí cumplan alguno de ellos (como la formación especializada reconocida) y, en el inicio de ese camino de acreditación, ya realicen diversas acciones dentro del deporte. Además, y como hasta la fecha no ha habido este reconocimiento profesional a través de la acreditación, actualmente hay muy pocos psicólogos/as acreditados como PEPD, aunque sí hay muchos con suficiente formación y experiencia avalada a través de una amplia y eficaz trayectoria psicológica en este deporte.

Quizás, en un futuro no muy lejano, sería adecuado plantear una sub-especialización de PEPD en fútbol, aunque lo realmente importante es que el trabajo de los profesionales de la Psicología que actúen en este deporte sean competentes, realicen eficientemente su función y se conviertan cada vez más en el profesional "mental" de referencia en un equipo técnico o club de fútbol por su verdadera valía profesional evidenciada con su trabajo (Peris-Delcampo, 2017b), para lo que planteamos los criterios anteriormente descritos de los que, como conclusión en este sentido, es necesario que PEPD adquiera unas competencias profesionales específicas para el fútbol.

\section{Competencias del psicólogo/a experto en fútbol}

Un/a profesional de la Psicología del Deporte que trabaje en fútbol debe ser capaz de adaptarse a sus circunstancias específicas para ofrecer soluciones óptimas a las necesidades psicológicas de un equipo de fútbol y de todos sus componentes. Ha de tener formación en Psicología del Deporte (saber), la adecuada actitud para el desempeño profesional (querer), y además saber desempeñar de forma práctica su labor (saber hacer) para ser eficaz y eficiente.

Es necesario, por tanto, que adquiera unas competencias profesionales, entendiéndolas como una capacidad aprendida para realizar adecuadamente una tarea, función o rol (Roe, 2002), o un "conjunto de comportamientos observables que estén causalmente relacionados con un desempeño bueno o excelente en un trabajo concreto y en una organización concreta" (Pereda y Berrocal, 1999, p. 21), cuyos aspectos fundamentales son el poseer unos conocimientos (saber), desarrollar unas habilidades (saber qué hacer) y tener las actitudes (querer hacer) necesarias para hacerlas adecuadamente (Levy-Leboyer, 1997), siendo además necesario demostrar su valía en un puesto o tarea concreta (Peiró, 2010).

En la actualidad contamos con una descripción de las competencias profesionales necesarias para la Psicología del Deporte definidas desde la División de la Actividad Física y el Deporte del Consejo General de la Psicología de España (PACFD), en línea con las establecidas para toda Europa y que en gran medida provienen de aquéllas (Cantón y Pérez, 2017). Siguiendo a este trabajo y pensando en las necesidades competenciales del psicólogo/a aplicado a un deporte como el fútbol, se pueden destacar de manera específica y ajustada a este deporte, las siguientes diez capacidades que como propuesta entendemos debería dominar este profesional para hacer un trabajo óptimo en este deporte.

\section{Competencia 1: Manejo y uso de habilidades psicológicas básicas relacionadas con la Psicología del Deporte}

El especialista deberá tener los conocimientos psicológicos necesarios para poder realizar sus actuaciones profesionales en el fútbol. Esto es, a modo más general, deberá conocer las técnicas o estrategias psicológicas, su fundamento y cuándo saber aplicarlas, en función de determinadas circunstancias.

Por ejemplo, deberá saber establecer adecuadamente los objetivos, diversas técnicas de control de la activación, cómo regular el estrés o fomentar la autoconfianza... y además conocer sus fundamentos psicológicos para que, cuando las utilice, su aplicación sea de calidad, eficaz y eficiente.

\section{Competencia 2: Conocimiento del entorno de aplicación relacionado con el fútbol}

El fútbol es un deporte diferenciado de todos los demás, sobre todo por la repercusión mediática así como por el número de practicantes que tiene a lo largo de todo el mundo. Esto hace que existan varias metodologías de trabajo, formas de interpretar este deporte e incluso entornos distintos donde el profesional de la psicología debería intervenir. Por ejemplo, existen diferencias respecto al entorno de intervención de un club de fútbol base de una ciudad pequeña, o la de un equipo profesional de fútbol; o también en función de la metodología de trabajo que utilice el propio club o equipo. 
A rasgos generales, y de modo más clásico, existe una metodología analítica (véase Álvarez del Villar, 1985) donde se trabaja cada aspecto del entrenamiento por partes, es decir hay un apartado para la técnica, otro para la táctica, la física y para la psicológica (si es que se destina tiempo para ello).

Después apareció la metodología globalizada o integrada (Seirulllo, 1999), donde, a través de varios ejercicios, se trabajan varios aspectos del entrenamiento (técnica, táctica y física; a veces psicológica) entendiéndolos como partes separadas que se integran en un solo ejercicio.

Una perspectiva relacionada y todavía más reciente es la que está basada en la especificidad del juego, desde una perspectiva más sistémica; donde se entiende el fútbol como un todo, que todo está interrelacionado y donde sólo se habla de partes diferenciarlas para estudiarlas mejor, pero no se puede trabajan ninguna de ellas excluyéndola del todo (Tamarit, 2013)

Obviamente, en función de cada metodología de trabajo (y cada entorno concreto), la actuación del psicólogo aplicado al fútbol será distinta. Por ejemplo, si tiene que realizar un trabajo de intervención directa desde la perspectiva analítica, PEPD tendrá su espacio y su tiempo para hacerlo, bien sea en el propio terreno de juego o en una sala (o vestuario) aparte; en cambio si la metodología de trabajo es globalizada o similar, incorporará objetivos y contenidos psicológicos a las sesiones de entrenamiento para desarrollar ciertas variables "mentales", junto con otras técnicas, tácticas, condicionales...

Además, existen diferencias también en relación al momento evolutivo, el nivel de los jugadores, las exigencias... Por ejemplo no es lo mismo trabajar en un equipo de fútbol base alevín de barrio, que en un club de élite de la misma edad. Ni tampoco en un primer equipo de una de las mejores ligas, que en otro de categoría regional... Existen aspectos diferenciales de cada entorno donde se deberá saber manejar. (Peris-Delcampo, 2017b).

El profesional de la Psicología en el fútbol deberá ser competente en conocer qué tipo de entorno relacionado con el fútbol es en el que se realiza su desempeño profesional, y cómo adaptar su rol profesional, estrategias, aptitudes... a cada entorno concreto. Deberá saber, por ejemplo, cómo intervenir desde la figura de un "psicólogo de club" en el que sólo hay un profesional para varios equipos, entrenadores... o como miembro del cuerpo técnico (donde es "un técnico más" que aporta "su parte").

\section{Competencia 3: Detectar necesidades específicas de cada entorno de aplicación}

De entrada, parece lógico pensar que no existe la misma "demanda psicológica" en un equipo de fútbol base de barrio con unos objetivos exclusivamente formativos, que en un club profesional, donde es posible que tenga objetivos diferentes, más centrados en el rendimiento deportivo y en el de formar jugadores de un perfil determinado pensando en que puedan llegar al primer equipo. Tampoco son exactamente los mismos objetivos de trabajo psicológico en el fútbol base que en un equipo de fútbol que pretenda, por ejemplo, subir de categoría. También es necesario adaptar el trabajo psicológico en función de otros parámetros de la situación sobre la que se interviene, como las diferencias en la metodología de trabajo, de las experiencias previas con psicólogos del deporte, de lo que realmente se espera de este profesional, de las posibilidades reales, etc.

Desde este punto de vista (Peris-Delcampo, 2017b), una buena manera de enfocar la labor profesional consiste en establecer a priori las necesidades psicológicas del deporte en general y del fútbol en particular, pensando en el manejo adecuado de las variables psicológicas relacionadas con el rendimiento deportivo aplicadas al fútbol (Almeida, 2010; Buceta, 2016; Peris-Delcampo, 2008, 2016, 2017b), entre las que destacan algunas como la autoconfianza, control del estrés, motivación, nivel de activación, atención/concentración, cohesión de equipo y comunicación; aunque, por ejemplo, en el caso de los equipos con objetivos más formativos o de base, estarán más orientados al desarrollo personal integral del joven deportista (y no tanto al aspecto esencialmente de rendimiento futbolístico).

Sea cual fuere el criterio de PEPD, es necesario que defina las necesidades psicológicas concretas para después planificar el trabajo psicológico a realizar y establecer los criterios y procedimientos de valoración.

\section{Competencia 4: Planificar, establecer objetivos y estrategias ajustadas a las necesidades detectadas}

Sobre la base de las necesidades psicológicas detectadas, el profesional de la Psicología del deporte aplicado al fútbol deberá ser capaz de realizar una planificación de su trabajo, teniendo en cuenta las posibilidades reales que le permitan el equipo, club, organización... En línea con lo planteado por diferentes especialistas (Arbinaga y Caracuel, 2011) y en relación a esta competencia, una vez detectadas las necesidades psicológicas del club/equipo deberá tener en cuenta:

- Los objetivos generales del club/equipo.

- Las posibilidades de intervención psicológica atendiendo a las características específicas del club, equipo, jugadores, técnicos...

- Valorar cuáles son las mejores opciones para que tenga un "mayor impacto" psicológico sin tocar demasiadas cosas. 
- Preparar posibles dificultades que se puedan presentar.

- Preparar la manera de evaluar el trabajo realizado.

Es decir, buscará la manera de realizar una planificación de la intervención psicológica aprovechando las características de la propia organización o club. Sólo añadirá más elementos de intervención si, al detectar las necesidades, no encuentra una buena manera de intervenir si no es añadiendo nuevos elementos de intervención (Peris-Delcampo 2017a, 2017b).

Por ejemplo, si detecta que la necesidad principal de un equipo de fútbol es el control de la "ansiedad" del equipo en los momentos finales del encuentro (manejo de la variable psicológica "control del estrés"), podría crear situaciones en los entrenamientos en los que se simulen las condiciones de competición fomentando la percepción de control de los futbolistas, en lugar de dar una charla a los jugadores explicando cómo funciona la ansiedad.

También, por ejemplo, en una escuela deportiva donde detecte que existe un grupo de padres problemáticos, valorará el hacer una intervención global sobre el entorno, en lugar de intervenir y señalar directamente a esos padres que pueden comportarse "mal" en un momento dado.

Un ejemplo de planificación general desde la perspectiva de la variable psicológica es la mostrada en la Tabla 2, en la que se determinan los indicadores de un buen y un mal funcionamiento de la variable "control del estrés" en una situación de partido junto con posibles estrategias psicológicas a aplicar.

Como propuesta de puntos clave a tener en cuenta por PEPD para la planificación del trabajo psicológico en fútbol, planteamos los siguientes siete pasos a seguir para realizar una adecuada planificación psicológica en un deporte como el fútbol (adaptados de Buceta, 1999; y Peris-Delcampo, 2016):

1. Detección de la demanda especifica: PEPD deberá ser capaz de, por un lado escuchar lo que se le requiere, también ofrecer aquello que puede ofrecer (cuando sea el momento) y, además, detectar necesidades psicológicas que no se manifiestan (pero que están ahí) que PEPD puede hacer frente, y que quizás no se le "pide" directamente (demanda encubierta).

2. Análisis del entorno donde es posible intervenir: cada contexto es diferente a los demás, por lo que es necesario analizar las características particulares del entorno que tiene que ver con la filosofía del club, el tipo de futbolistas, la metodología de trabajo del cuerpo técnico, los tiempos, espacios donde puede trabajar, las experiencias previas de trabajo psicológico..

3. Conocimiento de los objetivos deportivos: el trabajo psicológico debe ir ligado a los objetivos deportivos del club, por lo que PEPD deberá conocerlos e intentar incorporar y relacionar los objetivos psicológicos relacionados con la demanda y en función de las posibilidades, a los deportivos, para hacer un trabajo lo más ajustado al entorno posible.

4. Análisis de las posibilidades de intervención: una vez definidos los objetivos más adecuados a ese equipo, club, institución... PEPD valorará cuáles son los agentes sobre los que intervenir y de qué manera hacerlo. Es decir, estimará si es adecuado realizar formación a técnicos, si trabajar conjuntamente con el cuerpo técnico, realizar intervenciones grupales con futbolistas, modificar el entorno en función de las posibilidades...

5. Planificación de los objetivos psicológicos incluidos en la planificación psicológica global: PEPD buscará la manera de

Tabla 2: Ejemplo de variable psicológica en el fútbol, con los indicadores de mal y buen funcionamiento en el fútbol y posibles estrategias para intervenir sobre ella

\begin{tabular}{|c|c|c|c|c|}
\hline & Definición & $\begin{array}{l}\text { Indicadores de mal } \\
\text { funcionamiento }\end{array}$ & $\begin{array}{l}\text { Indicadores de buen } \\
\text { funcionamiento }\end{array}$ & $\begin{array}{l}\text { Posibles estrategias de } \\
\text { trabajo para controlar el estrés }\end{array}$ \\
\hline 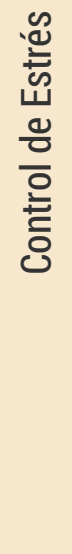 & $\begin{array}{l}\text { Movilización de } \\
\text { recursos fisiológicos y } \\
\text { psicológicos ante una } \\
\text { situación que se percibe } \\
\text { como amenazante. }\end{array}$ & $\begin{array}{l}\text { - Sensación de tensión } \\
\text { muscular. } \\
\text { - Mirada tensa. } \\
\text { - Precipitación. } \\
\text { - Mensajes inespecíficos e } \\
\text { inadecuados. } \\
\text { - Bloqueo. } \\
\text { - Déficit atencional. }\end{array}$ & $\begin{array}{l}\text { - Estado de funcionamiento } \\
\text { óptimo. } \\
\text { - Superación eficiente de } \\
\text { errores. } \\
\text { - Toma la iniciativa. } \\
\text { - "Realiza su trabajo" } \\
\text { - Sensación de "Flow". }\end{array}$ & $\begin{array}{l}\text { - Reducir incertidumbre. } \\
\text { - Entrenar situaciones } \\
\text { estresantes. } \\
\text { - Prevención de dificultades. } \\
\text { - Establecimiento de objetivos. } \\
\text { - Disminución cognitiva de la } \\
\text { amenaza. } \\
\text { - Control de situaciones } \\
\text { potencialmente estresantes. } \\
\text { - Preparación adecuada de la } \\
\text { competición. }\end{array}$ \\
\hline
\end{tabular}


optimizar el trabajo psicológico integrándolo en la planificación global deportiva, para lo que incorporará los objetivos meramente psicológicos en el conjunto de los deportivos. Por ejemplo, añadirá a los técnico-tácticos-físicos el manejo de variables psicológicas o en la formación de entrenadores algunos relacionados con las competencias mentales. Obviamente, el tener en cuenta las propias características de la organización o club, junto con la metodología de trabajo es fundamental en este punto.

6. Aplicación de las estrategias psicológicas y el plan de acción: llega el momento de buscar, decidir y preparar las estrategias psicológicas más adecuadas para ese equipo, club... entorno particular de aplicación, en función de los aspectos anteriores. PEPD deberá ser creativo, eficiente y adaptarse a las necesidades reales con estrategias psicológicas útiles (fundamentadas desde la psicología) buscando soluciones útiles.

7. Valoración y seguimiento: el cómo evaluar y hacer un seguimiento sobre el trabajo realizado por parte de PEPD es fundamental para asegurarse la adecuada realización del mismo. La manera de hacerlo ya es otra cosa, depende mucho de los puntos anteriormente mencionados: deberá estar muy adaptada a las características de la organización con la que se trabaje, objetivos planteados... PEPD utilizará en este sentido herramientas de observación, entrevistas "informales" con futbolistas, técnicos... percepción subjetiva del trabajo realizado, diferentes gráficos (si es posible)... aquellos elementos que le ayuden a evaluar su trabajo en ese entorno con unas características particulares, teniendo en cuenta no ser excesivamente intrusivo, desde la "normalidad" habitual de la organización, club, equipo... en el que trabaje.

\section{Competencia 5: Trabajar en equipo}

El psicólogo del deporte deberá, por un lado ser competente en contribuir a generar un equipo de calidad con buen rendimiento (para poder asesorar/intervenir con otros a que sean capaces de hacerlo), y por otro funcionar dentro de un equipo de trabajo en un rol determinado y con unas condiciones determinadas.

Por ejemplo, si forma parte del cuerpo técnico junto con profesionales de las ciencias del deporte y otros técnicos, deberá funcionar de manera óptima en relación a las demandas de ese grupo de trabajo; y también tendrá que dominar estrategias de funcionamiento de los equipos, para así favorecer que el equipo deportivo sea óptimo, realizando estrategias por sí mismo o bien asesorando al entrenador para que "cree equipo".

\section{Competencia 6: Desarrollo de la individualidad adaptada al entorno}

Aunque el fútbol es un deporte colectivo, es necesario prestar la suficiente atención a las características individuales de cada futbolista y de cada agente implicado en el club o equipo de fútbol. Es decir, PEPD debe ser capaz de desarrollar su trabajo desde el entorno global, prestando la suficiente atención a las características individuales de los agentes implicados, entendiendo a cada persona como única que está y funciona en un club o equipo determinado.

Por ejemplo, a la hora de trabajar con un equipo de fútbol podrá realizar, junto con el entrenador, diferentes estrategias para el control de la activación del equipo; mientras que a nivel individual atenderá cada caso que necesite manejar estrategias específicas para regular el nivel de activación individual que cada cual requiera.

También será capaz de ajustar los objetivos individuales (y la manera de trabajarlos) a cada futbolista en función de las características y demandas específicas y un largo etcétera.

\section{Competencia 7: Autocontrol y desarrollo emocional}

La propia autogestión de PEPD en el fútbol es fundamental para que pueda realizar su trabajo, sobre todo en los momentos más delicados y donde se precisa que PEPD esté en condiciones óptimas para dar "lo mejor de sí". Es decir, PEPD debe autorregularse para, sobre todo cuando más se le necesita, estar en su estado óptimo de rendimiento profesional y así aplicar mejor sus conocimientos psicológicos. Además es especialmente necesario que esta competencia se desarrolle, ya que se puede dar el caso en el que, por ejemplo el cuerpo técnico en su totalidad (incluido PEPD) sienta la presión por resultados inmediatos y sea el profesional de la psicología el que deba, en primer lugar "estar bien" (autorregularse) y después ayudar a que los demás "estén bien" para hacer el trabajo de la mejor manera posible.

\section{Competencia 8: Búsqueda de soluciones útiles}

En lugar de centrarse en aplicar determinadas técnicas solo por costumbre o porque aparezca en algún manual de trabajo, debe buscar la forma de dar respuesta, de la mejor manera, a aquellas necesidades mentales que detecte. Es decir, estará centrado en encontrar esa solución óptima, aplicando la mejor estrategia psicológica que se 
demande en ese momento y resolver esa situación. Debe ser capaz de adaptar los instrumentos, técnicas y procedimientos estandarizados y bien fundados a la casuística y necesidades concretas de cada caso. Esta es probablemente una de las funciones más importantes y que requiere una extensa y profunda capacitación profesional en Psicología.

Por ejemplo, cuando prepare, junto con el primer entrenador, la rueda de prensa o la charla siguiente a la finalización de un partido, elegirán conjuntamente pocos pero potentes mensajes en la línea de los objetivos planteados.

También, cuando se pretenda mejorar la autoconfianza respecto a una situación de juego (por ejemplo córneres en contra), puede plantear una intervención junto con el cuerpo técnico donde se trabajen y refuercen conductas concretas que favorezcan el éxito en esas situaciones, integrado dentro del trabajo general del equipo.

Es posible, además, que PEPD colabore en la realización de tareas de entrenamiento donde se trabajen específicamente objetivos psicológicos además de otros más "clásicos" del fútbol, como se observa en la Figura 1.

\section{Competencia 9: Discreción, confidencialidad y compromiso ético}

El código deontológico del Consejo General de la Psicología de España (CGPE, 2015) regula el funcionamiento del profesional de la psicología y también lógicamente de sus especialidades, si bien con los matices propios de éstas, que seguramente convendría compilar en algún tipo de documento que facilitara su uso específico. Acogerse a él no sólo favorece el buen funcionamiento psicológico de las personas con las que trabaja, sino que además asegura, a los contratantes, que los expertos en psicología del deporte actuarán con profesionalidad en beneficio de los futbolistas, técnicos, dirigentes... de todos los agentes implicados en el fútbol.

Esto implica discreción (no hablar de aspectos íntimos respecto a lo que se trabaja), confidencialidad (no decir con quién se trabaja; aunque si es público puede decir que tiene contrato con un equipo o un club) y mantener un compromiso ético con honestidad (hacer lo mejor para cada persona con la que trabaja). Estos tres aspectos generan un clima de confidencialidad y de confianza para que futbolistas, entre-

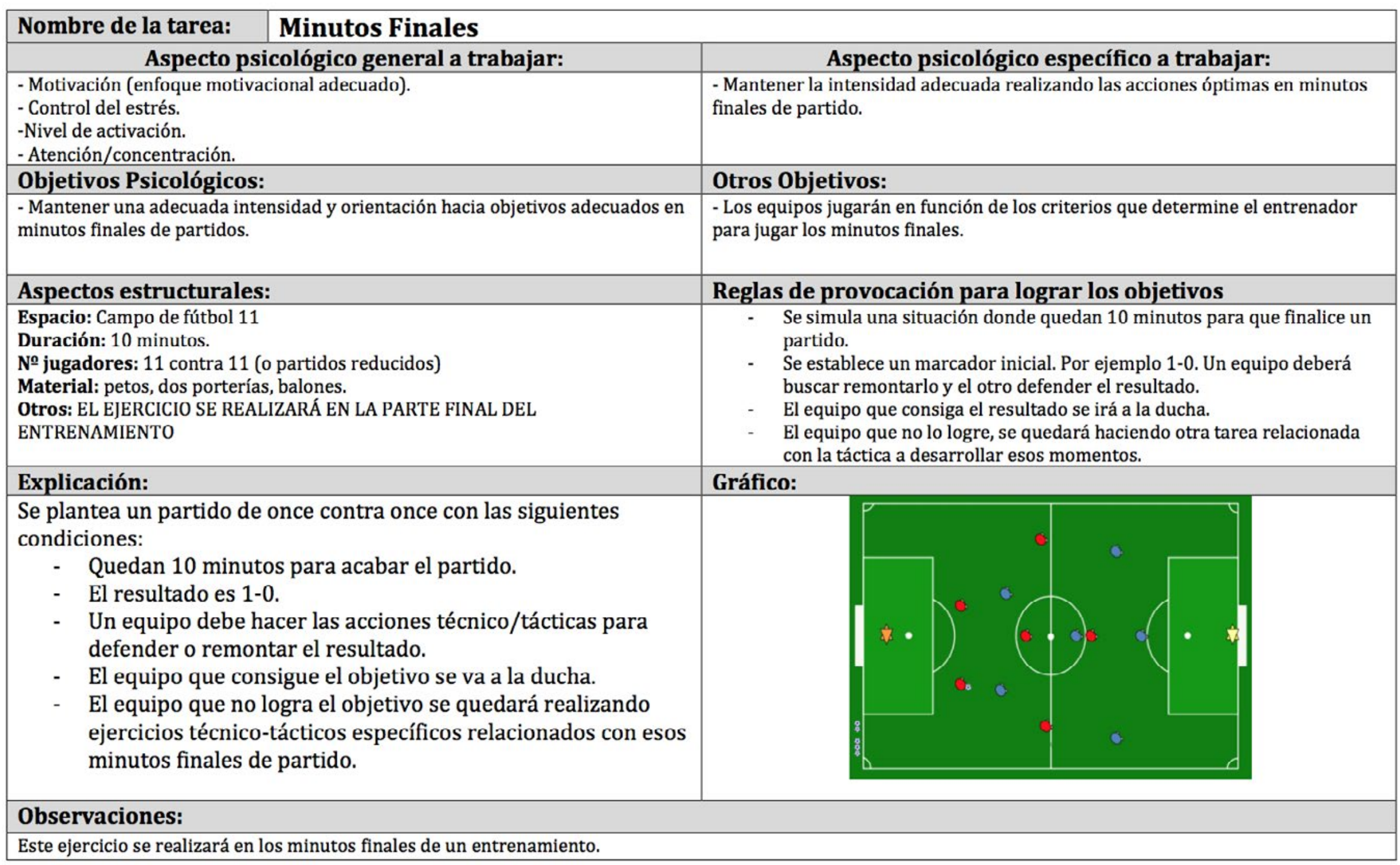

Figura 1. Tarea de entrenamiento para trabajar los minutos finales (Fuente: elaboración propia). 
nadores, técnicos y demás entiendan (de verdad) que lo que van a contarle y trabajen con PEPD se quede ahí; y así sea PEPD el único profesional que pueda trabajar con garantías los aspectos psicológicos más personales e íntimos legalmente protegidos.

Por ejemplo, un futbolista que está preocupado porque tiene una molestia leve en una pierna y no ha dormido bien la noche anterior por esa causa no le va a contar al entrenador ni a otro ayudante su situación si tiene mucho interés en jugar un partido importante que se disputará en un corto periodo de tiempo. En cambio, si PEPD ha generado un clima de confianza, donde el entrenador y el futbolista saben que lo que hable un jugador con PEPD "se queda ahí"; este deportista podrá trabajar este aspecto con PEPD para que éste le ayude a tomar la mejor decisión, bien sea decírselo al entrenador, asumir las consecuencias y, sobre todo, manejar los aspectos cognitivos relacionados con esa situación que le generan preocupaciones y le alejan de su mejor funcionamiento.

PEPD debe crear y mantener su credibilidad profesional para poder realizar un trabajo completo con todos los agentes que quieran trabajar con él. Y eso tiene que ver con que sea discreto y mantenga la confidencialidad y el compromiso ético, junto con el primer y más importante principio del código que es poner por encima de todo el bienestar y la salud de la persona.

\section{Competencia 10: Márketing adecuado}

Dentro de las aparentes limitaciones deontológicas de, por ejemplo tener que mantener la confidencialidad, el psicólogo/a del deporte tiene múltiples oportunidades para mostrar que es un profesional válido para un deporte como el fútbol y la mejor manera de hacerlo es haciendo un trabajo útil, profesional y "estando en todo momento en su lugar". Esto significa por ejemplo, no hablar de los futbolistas y otros agentes con los que trabaja; limitar y cuidar mucho sus apariciones en público cuando está contratado con un club, teniendo muy en cuenta las recomendaciones del departamento de comunicación (si es que la entidad lo posee) y dar una imagen pública seria, "hablando de psicología aplicada al fútbol" sin buscar el protagonismo intentando "colgarse medallas" que no le corresponden. Es más, debe mantenerse quizás "en segundo plano" dejando que sean otros los que tengan más visibilidad. De hecho, hay toda una normativa legal sobre protección de datos personales que obliga a la discreción y que, como poco, requiere de un consentimiento informado del cliente para poder mencionarlo.

Ahora bien, teniendo en cuenta estos aspectos, el profesional de la Psicología del Deporte deberá saber mostrar su trabajo con inteligencia y eficacia, recordando además que el "boca a boca" es una gran tarjeta de presentación.

En ocasiones, ha habido psicólogos/as que, en el momento en que se les ha contratado para un club, ha realizado intervenciones en medios de comunicación donde incluso parece que han sido los "salvadores" de un equipo o que alguna acción puntual suya ha resultado decisiva en la obtención de un resultado. Esto, además sabiendo que el éxito o el fracaso es un trabajo de todos, es contraproducente ya que, entre otras cosas, el entrenador, ayudantes o incluso los jugadores pueden verse amenazados al perder el protagonismo "por culpa" de esos psicólogos/as.

Otra cosa distinta es cuando, por ejemplo, invitan a un PEPD a un medio de comunicación para que "resuelva" una situación "mental" de un equipo. En esta ocasión, si dicho PEPD no pertenece a ningún club, podrá opinar libremente, entendiendo que no tiene toda la información y hablando de aspectos psicológicos en un lenguaje entendible, siendo realista e intentando no dejarse llevar por el espectáculo y no caer en triunfalismos excesivos. Estas son buenas oportunidades para hacer entender que la psicología es una ciencia, que es aplicable y que no son "cosas raras", sino que forma parte de la tecnificación del fútbol en el día a día.

Un aspecto cada vez más importante en relación al márketing personal del profesional de la psicología que trabaja en fútbol es el manejo y buen uso de las redes sociales; donde PEPD utilizará el mismo estilo de funcionamiento que en otros entornos, aun sabiendo la inmediatez y características particulares de cada red social para, a la vez que ofrecer una imagen seria, respetar el código deontológico y, sobre todo la confidencialidad de los "clientes".

Es una opinión muy compartida por los profesionales del área, que seguramente lo mejor en un club o un equipo es que PEPD esté en segundo plano, manteniendo la confidencialidad realizando un trabajo serio y buscando el beneficio de los agentes con los que trabaja. Esa es la mejor manera de mostrar su trabajo.

\section{Principios generales de actuación}

Partiendo del perfil profesional sugerido y las competencias establecidas, a continuación se presentan ocho principios generales a tener en cuenta por un PEPD que trabaje en fútbol (adaptados de Peris-Delcampo, 2017a, 2017b):

1. Optimización: PEPD aplicado/a al fútbol es capaz de ser eficiente, interviniendo de forma no extensiva o intrusiva pero buscando el máximo impacto psicológico en las personas con las que actúa. Es decir, optimiza su trabajo. 
Su principal foco de atención está en las personas sobre las que sus actuaciones tienen efecto psicológico, buscando adaptar su trabajo (como se ha señalado) a las condiciones de la propia institución, club o equipo. Se trata de aplicar la Psicología para lograr el máximo beneficio con el menor gasto de recursos (sin escatimarlos, eso sí) y con el máximo fundamento psicológico aplicado.

2. Intervenir no es investigar: la Psicología es una ciencia y, como tal, utiliza el método científico para recoger datos, sacar conclusiones y generar respuestas a distintas preguntas en principio aplicadas. La investigación, a través del método científico, necesita recoger datos, con unas condiciones determinadas, para después analizarlos y sacar las conclusiones que después serán publicadas en alguna revista científica. La buena investigación es necesaria para el avance de la ciencia. Cuando PEPD interviene en fútbol (en cualquiera de las posibilidades señaladas en puntos anteriores), detecta necesidades psicológicas y aplica de la mejor manera que cree oportuno, aquellas estrategias para beneficiar y mejorar el funcionamiento de futbolistas, técnicos, padres y madres, dirigentes... La adecuada intervención psicológica es necesaria para el avance de la psicología aplicada y se nutre de los conocimientos científicos que se generan desde la investigación. Pero estos dos procesos no son necesariamente lo mismo (Cantón, 2010b). Los objetivos para cada uno de los casos son diferentes y la manera de actuar de los psicólogos que realizan cada una de las dos acciones también es, en la gran mayoría de los casos, distinta. PEPD debe ser consciente de esta diferenciación y dejar bien claro que su prioridad es el funcionamiento psicológico de los diversos agentes sobre los que interviene y no tanto el recoger datos para investigar, aun sabiendo que en algunos casos (explicando bien la cuestión a los deportistas) es posible combinar ambos tipos de acciones. Es importante, además, que sobre todo los responsables del fútbol entiendan esta diferencia, para evitar la negativa e inadecuada percepción de que los psicólogos "sólo pasan test" o que la intervención psicológica no produce resultados visibles, porque algunos psicólogos/as han ido a un club de fútbol sin dejar clara esta diferenciación. Cada cosa es necesaria y hay que diferenciarlas tanto por PEPD como por los distintos agentes del fútbol.

3. Anticiparse es mejor que resolver situaciones comprometidas: una buena manera de solucionar problemas es evitar que ocurran, es decir, prevenir. PEPD es un experto/a en comportamiento humano y en análisis funcional, por lo que debe ser capaz de detectar situaciones (estímulos antecedentes) que pueden provocar dificultades o problemas que habrá que subsanar. Es adecuado, por tan- to, que PEPD tenga en cuenta esta cuestión y busque la manera de anticipar posibles dificultades "mentales" aplicando estrategias para que el funcionamiento de futbolistas, técnicos y demás sea bueno, velando por que no aparezcan dificultades al respecto.

4. Preparar el terreno para poder intervenir: no es lo más habitual todavía que un futbolista quiera trabajar con el psicólogo/a de manera individual cuando éste ha llegado por primera vez a un equipo o un club de fútbol. También, en clubes de fútbol suele ocurrir que haya entrenadores que trabajen con facilidad con el psicólogo, mientras que otros tengan cierta reticencia para trabajar con ellos. Conviene que PEPD aprenda a preparar el terreno (Buceta, 1999), es decir, realizar una serie de acciones para que realmente esos agentes del fútbol quieran trabajar directamente con PEPD; por ejemplo, estando presente vestido con la ropa del club como un técnico más, aprovechando pequeños contactos sociales para establecer cierta relación, manteniendo un comportamiento profesional en el que se muestre como persona que hace un trabajo confidencial, etc.

5. Elegir bien los momentos de intervención: una de las grandes cualidades del buen profesional PEPD en fútbol es el observar desde un lugar y papel adecuado, para detectar esos momentos asociados a esas necesidades psicológicas donde la intervención sea más conveniente, eso sí, siempre pensando en el beneficio psicológico de futbolistas, técnicos y familias. También, para intervenir con eficacia, es necesario el haber generado las condiciones donde las personas con las que se va a intervenir estén dispuestas a hacerlo. Y hacerlo en el momento adecuado y de manera óptima.

6. Profesionalidad: el psicólogo/a del deporte en el fútbol puede realizar bien su trabajo cuando funciona en base al código deontológico que, además, hace que cree las condiciones para que su función la realice con éxito. Existen, en relación a este apartado, tres aspectos fundamentales que debe respetar para realizar su trabajo con profesionalidad: confidencialidad, honestidad y "saber estar" manteniéndose en todo momento en su papel y rol profesional.

7. "Hablar de Fútbol", desde la Psicología aplicada al fútbol: el psicólogo/a del deporte debe dominar conocimientos y estrategias psicológicas y manejar adecuadamente estos conceptos; y también deberá adaptar su lenguaje, su forma de actuar y manera de comunicarse a los jugadores, técnicos y otros agentes implicados. No se trata de tener conocimientos extensos sobre fútbol, al igual que lo podría tener un entrenador; sino que sea capaz de utilizar el mismo lenguaje que el resto del cuerpo técnico para, eso sí, aplicar la psicología de manera óptima. 
8. Soluciones: la orientación de la intervención psicológica del profesional que trabaja en fútbol, debe estar centrada en soluciones (y no tanto en tratar "sólo problemas" o "aplicar técnicas" que solo sobre el papel tienen un resultado satisfactorio). Es decir, debe buscar la manera de encontrar esa solución óptima, con contenido psicológico, ofreciendo una solución útil.

\section{Discusión}

La función del psicólogo/a del fútbol (esperemos que muy pronto podamos hablar de que todos son Psicólogos Expertos en Psicología del Deporte o PEPD, en el fútbol, o estén en el proceso de obtención de esta acreditación) es cada vez más demandada y cada vez más se cuenta con estos profesionales en los equipos y clubes de fútbol. Eso es gracias al buen trabajo de muchos psicólogos/as que durante años se han mostrado útiles, eficaces y eficientes, resolviendo las diferentes necesidades de todos los estamentos que conforman el mundo del fútbol; "superando" la no tan buena imagen de otros psicólogos o "similar" que no han dejado una buena impresión ni la percepción de haber realizado un buen trabajo "mental".

Afortunadamente, el perfil profesional del psicólogo/a del deporte está definido de manera aplicada y, cada vez más, los distintos agentes del deporte buscan no sólo a "cualquier" psicólogo/a, sino a profesionales de la Psicología que estén especializados en Psicología del Deporte y, sobre todo que sepan dar respuesta a las diferentes necesidades "mentales" que surgen en el deporte actual. Lamentablemente, esto no ocurre en todos los casos y existe un gran trabajo por hacer para que realmente los distintos agentes del fútbol perciban de manera clara que el "profesional mental" en el deporte (y en especial el fútbol) es un/a psicólogo/a especializado en Psicología del Deporte; y no "cualquier psicólogo/a" sin la adecuada especialización y competencias y mucho menos otros "pseudoprofesionales" que pretenden ocupar este espacio.

Obviamente, aunque ya existe un gran camino recorrido, aún queda mucho por hacer y donde seguramente la acreditación profesional del psicólogo/a especialista en psicología del deporte y quizás una subespecialización en fútbol, junto con el buen y profesional trabajo de los distintos psicólogos/as del fútbol hagan que los diferentes agentes de este deporte busquen a PEPD del fútbol cuando demanden un profesional "mental" y eso, a su vez, sea sinónimo de calidad.

Por último, destacar la necesidad de cada psicólogo/a de desarrollar las competencias necesarias para un exce- lente ejercicio de la profesión, así como tener presente los principios generales de actuación que guíen su desempeño, tal y como se ha expuesto en el presente trabajo. Por ejemplo, la diferente oferta formativa en Psicología del Deporte debería de tener presente estas cuestiones, al igual que el entrenamiento en competencias personales (p. ej., trabajo en equipo, responsabilidad y comunicación) que favorecerá la labor profesional del PEPD en el fútbol.

\section{Referencias}

Almeida P. (2010). Variables psicológicas y rendimiento deportivo en fútbol. En J. M. Buceta, y E. Larumbe, (Eds.) Experiencias en Psicología del Deporte. (pp. 478-488). Madrid, España: Dykinson.

Álvarez del Villar, C. (1985). La preparación física del futbol basada en el atletismo. Madrid, España: Editorial S.L. GYMNOS.

Arbinaga y J. C. Caracuel (2011). Intervención psicológica en actividad física y deportes minoritarios. Madrid, España: Editorial Psimática.

Buceta, J. M. (1999). Psicología: Curso Nivel 3. Madrid, España: Real Federación Española de Fútbol.

Buceta, J. M. (2016). Variables psicológicas relacionadas con el rendimiento físico y deportivo. Madrid, España: Dykinson.

Cantón, E. (2010a). La Psicología del Deporte como profesión especializada. Papeles del Psicólogo, 37, 237-245.

Cantón, E. (2010b). Un caso paradigmático de la encrucijada actual en Psicología del Deporte: el trabajo e investigación en motivación. Informació Psicològica, 100, 24-36.

Cantón, E. (2016). La especialidad profesional en psicología del deporte. Revista de Psicología Aplicada al Deporte y el Ejercicio Físico, 1, E2. http://dx.doi.org/10.5093/rpadef2016a2

Cantón, E. y Pérez, E. (2017). Cómo ser competente como profesional de la psicología del deporte. Formación Continuada a Distancia del Consejo General de Psicología de España (FOCAD).

Consejo General de la Psicología de España (CGPE, 2015). Código deontológico del Psicólogo. Recuperado de: https://www.cop. es/index. php?page=CodigoDeontologico

Consejo General de la Psicología de España (CGPE, 2018). Comisión Nacional de Acreditación Profesional. Recuperado de: http:// www.acreditaciones.cop.es/

García-Barrero, J. y Llames, R. (1992). Preparación Psicológica de la Selección Olímpica de Fútbol. Revista de Psicología del Deporte, 2, 63-67.

Garcia-Naveira, A. y Jerez, P. (2012). Departamento de psicología del club Atlético de Madrid: filosofía, programación y desempeño profesional en el fútbol base. Cuadernos de Psicología del Deporte, 12(1), 111-120. https://doi.org/10.4321/S157884232012000100010

Levy-Leboyer, C. (1997). Gestión de las Competencias. Cómo analizarlas. Cómo Evaluarlas. Cómo desarrollarlas. Barcelona, España: Ediciones Gestión 2000

Morilla, M., Pérez, E., Gamito, J., Gómez, M., Sánchez, J y Valiente, M. (2003). Planificación psicológica de la cantera del Sevilla 
F.C.S.A.D.: Organización, funcionamiento y programa deportivo-formativo. Cuadernos de Psicología del Deporte, 3(2), 17-30.

Navarrón, E., Godoy-Izquierdo, D., Vélez, M., Ramírez-Molina, M. y Jiménez-Torres, M. (2017). Implementación de una intervención psicológica en fútbol base, satisfacción subjetiva de los deportistas y experiencias de pasión, competencia percibida y compromiso deportivo en relación con la intención de práctica futura. Revista Iberoamericana de Psicología del Ejercicio y el Deporte, 12(1), 59-69.

Olmedilla, A., García, C. y Garcés de los Fayos, E. J. (1998). Un análisis del papel profesional del psicólogo del deporte desde la percepción del entrenador de fútbol. Revista de Psicología del Deporte, 7, 95-113.

Pereda, S. y Berrocal, F. (1999). Gestión de recursos humanos por competencias. Madrid, España: Centro de Estudios Ramón Areces.

Peiró, J.M. (2010). Competencias para el ejercicio profesional del Psicólogo. Formación Continuada a Distancia del Consejo General de Psicología de España (FOCAD).

Peris-Delcampo, D. (2008). El entrenamiento psicológico en fútbol: variables implicadas. Tui, España: MC Sports.
Peris-Delcampo, D. (2016). Nociones básicas de psicología del deporte y coaching para gestores de empresas deportivas. Almería, España: Círculo Rojo.

Peris-Delcampo, D. (Febrero, 2017a). El psicólogo deportivo: formación, acreditación, regulación y actividad profesional. Comunicación presentada en el XV Congreso Andaluz y II Luso-Andaluz de Psicología de la Actividad Física y del Deporte, Granada, España.

Peris-Delcampo, D. (Junio, 2017b). Intervención Psicológica en Academias de Fútbol Base. Sesión de cartel presentado en el II Congreso Internacional de Psicología y Fútbol de la Universidad de Santiago de Compostela y Federación Gallega de Fútbol. Santiago de Compostela, España.

Roe, R. A. (2002). What makes a competent psychologist? European Psychologist, 7, 192-202. https://doi.org/10.1027//10169040.7.3.192

Seirul-lo, F. (1999). Criterios modernos de entrenamiento del fútbol. Revista Training Fútbol, 45, 8-18.

Tamarit, X. (2013). Periodización táctica vs. Periodización Táctica. Valencia, España: Management for Business in Football. 